Proceedings of ASME 2011 5th International Conference on Energy Sustainability \& 9th Fuel Cell Science, Engineering and Technology Conference, ESFuelCell2011

August 7-10, 2011, Washington, DC, USA

ESFuelCell2011-54257

\title{
Through-Plane Gas Permeability of Proton Exchange Membrane Fuel Cell Gas Diffusion Layers
}

\author{
A. Tamayol \\ Mechatronic Systems Engineering, \\ School of Engineering Science, \\ Simon Fraser University, BC, \\ Canada \\ ali_tamayol@sfu.ca
}

\author{
F. McGregor \\ Mechatronic Systems Engineering, \\ School of Engineering Science, \\ Simon Fraser University, BC, \\ Canada \\ fraser_mcgregor@sfu.ca
}

\author{
M. Bahrami \\ Mechatronic Systems Engineering, \\ School of Engineering Science, \\ Simon Fraser University, BC, \\ Canada \\ mbahrami@sfu.ca
}

\begin{abstract}
Effects of mechanical compression and PTFE content on the through-plane gas permeability of gas diffusion layers (GDLs) of PEM fuel cells are investigated both experimentally and theoretically. A new test bed is designed and built which allows pressure drop and air flow rate measurement for various fibrous samples. The measured values are used to calculate the through-plane permeability. Various GDLs are obtained and tested over a wide range of PTFE content and compression ratio. The experimental data shows a reverse relationship between the through-plane permeability and both PTFE content and mechanical compression. An existing model for through-plane permeability of planar structures is revisited to develop a model that accommodates effects of PTFE content and GDL compression. The proposed model captures the trends of the experimental data for through-plane permeability, measured in the present study or reported by others.
\end{abstract}

Keywords: PEM fuel cell, Through-plane permeability; Gas diffusion layer; Mechanical compression; Experimental study; Modeling.

\section{Introduction}

Proton exchange membrane fuel cells (PEMFCs) have attracted much attention in the past decade as a promising candidate for clean power sources in automotive, electronic, portables, and stationary applications [1]. Challenges facing the commercialization of PEMFCs are reliability, cost, power density, performance, and membrane lifetime. Membrane electrode assembly (MEA) is the heart of a PEMFC and is composed of a membrane covered by catalyst particles sandwiched between two porous gas diffusion layers (GDLs) on the sides [1].

During operation, hydrogen and oxygen are consumed in the anode and cathode sides of the MEA, respectively; and water and heat are produced in the cathode side. As a result of the electrochemical reaction, an electrical current is generated from anode to cathode side via external electrical loads.

To maintain a PEMFC at its optimum operating condition, reactants should be transported towards the catalyst layer continuously, where the electrochemical reactions occur. The excess produced water and heat should be removed and electrons should pass from the bipolar plate towards the catalyst layer and vice versa [2]; the GDL plays a 
crucial role in all these functions, moreover, it provides mechanical support for the MEA. As a result, the transport properties of GDLs are extremely important in accessing the overall performance of PEMFCs. The mass transport loss, related to GDL permeability, is the major cause for limiting the maximum power density of a PEM fuel cell. As such, an in-depth knowledge of permeability of GDLs is important for optimizing PEMFCs operation [3-10].

In a PEM fuel cell stack, the cell components are compressed together to prevent any gas leakage from the system. The applied pressure changes the GDL thickness and its porosity (microstructure); this affects the transport properties of the GDL to a considerable extent. The gas permeability reduces as a result of the GDL compression while the thermal and electrical conductivities increase [5]. As such, a trade off exists in the design and optimization process of MEAs [9, 11]. Thus, model(s) that can predict the permeability of GDLs as a function of porosity is a necessity for designing high performance PEMFCs.

Water management is one of the current challenges in increasing power density of PEMFCs. Use of hydrophilic GDLs leads to membrane dehydration and blockage of the pores and as a result hindering of reactant access to catalyst layers [1214]. To prevent these issues, the GDL is commonly treated with hydrophobic Polytetrafluoroethylene (PTFE); this affects the porosity and as a result the air permeability of the treated GDL. The mass transfer rate and the overall cell performance change by adding PTFE [15]. Therefore, the effects of PTFE content on the through-plane permeability should also be considered in design and optimization process.

Generally, GDLs are porous media made of carbon fibers with diameter of 7-10 $\mu \mathrm{m}$ in the form of carbon papers or carbon cloths [16]. These materials have complex microstructure with random distribution of fibers which prevent from exact solution of flow-field and the permeability. As such, the permeability of GDLs has been mostly determined either experimentally or numerically.

There are few experimental studies available in the literature in which the permeability of GDLs has been investigated [2, 15-20]. Ihonen et al. [15] showed that a reverse relationship exists between the in-plane permeability and compression. Recently,
Feser et al. [2] studied the effects of mechanical compression and reported the in-plane gas permeability as a function of porosity for a carbon cloth, a non-woven carbon fiber GDL, and a carbon paper. In a similar work, Gostick et al. [16] measured the permeability of several commercial GDLs under various compressive loads and reported the in-plane permeability as a function of porosity. They also reported through-plane permeability of several GDLs. Recently, Tahler et al. [20] and Becker et al. [19] have measured the through plane permeability of compressed Toray carbon papers. Gurau et al. [18] measured in-plane and through-plane permeabilities of a carbon paper with various PTFE contents and reported a direct relationship between PTFE content and permeability. On the contrary, the experimental observation of Park et al. [21] showed a reduction of permeability by increasing PTFE content.

On the theoretical side, Lattice Boltzmann simulations of gas flow through several random fibrous structures were carried out by Vandoormaal and Pharoah [22] over the porosity range of $0.6<\varepsilon$ $<0.8$. Their numerical results suggested a significant impact of fiber orientations on the permeability even in a constant porosity. Based on the numerical results for structures with mixed fiber orientations, they proposed two correlations for in-plane and throughplane permeabilities of the considered structures; however, these correlations are not applicable to compressed or PTFE treated GDLs. Becker et al. [19] used 3D tomography to reconstruct a GDL and a numerically efficient pore morphology method to determine phase distributions and to deduce permeability, diffusivity and thermal conductivity as a function of the saturation under different compressive loads. Hao and Cheng [23] reconstructed carbon papers with different PTFE contents using a stochastic method and solved the pore-level flow by Lattice Boltzmann method.

Shi et al. [24] employed the fractal theory and developed models for permeability that accounted for the microstructures of GDLs in terms of two fractal dimensions and proposed a relationship for permeability as a function of tortuosity, fractal dimensions, pore area, pore size distribution, and effective porosity. Their model, however, requires several geometrical parameters (e.g. fractal parameters) that should be known beforehand. A similar approach was also adopted by He et al. [25]. 
Recently, Tamayol and Bahrami [26] have proposed a blending technique for predicting the in-plane permeability of GDLs. They treated the medium as a mixture of fibers parallel and perpendicular to the flow direction and estimated the permeability as a blend of the parallel and normal permeability of unidirectionally aligned fibers [27].

Through-plane permeability of uncompressed carbon papers can be estimated using the existing models for the permeability of planar fibrous structures. Based on the analogy between electrical and flow conductions, Tomadakis and Robertson [28] developed a model for permeability of randomly distributed overlapping fibers in composite reinforcements. Recently, Tamayol and Bahrami [29] used a scale analysis technique and related the permeability of fibrous media to the microstructure geometrical parameters including tortuosity, pore diameter, and porosity. They have successfully compared their model with experimental data collected from various sources for a variety of materials. However, the models of [28] and [29] do not include the effect of PTFE content and mechanical compression on the through-plane permeability.

As such, there is a need for a general model that can accurately predict the through-plane permeability of GDLs as a function of compression ratio and PTFE content. In addition, more experimental investigations are required to find effects of mechanical compression and PTFE content on the through-plane permeability of GDLS.

In this study, through-plane permeability of several compressed GDLs is measured experimentally. In addition, effect of PTFE content on the permeability is investigated. The model of Tamayol and Bahrami [29] is modified to accommodate the effects of mechanical compression and PTFE contents.

\section{Experimental Approach \\ 2.1 Tested samples}

The tested GDLs were purchased as Teflon treated carbon papers from different manufacturers. As a result of their similar production procedure, despite the different thicknesses, TGP-H-90 and 120 have similar microstructures. TGP-H-120 was obtained with $0,5,20$, and 30\% PTFE content while the tested TGP-H-90 have a 0\% PTFE content. Figure 1a Shows a SEM image of TGP-H-120 with
5\% PTFE content. The SGL Sigracet 10AA, shown in Figure 1b, was obtained with 0\% PTFE content.

(a)

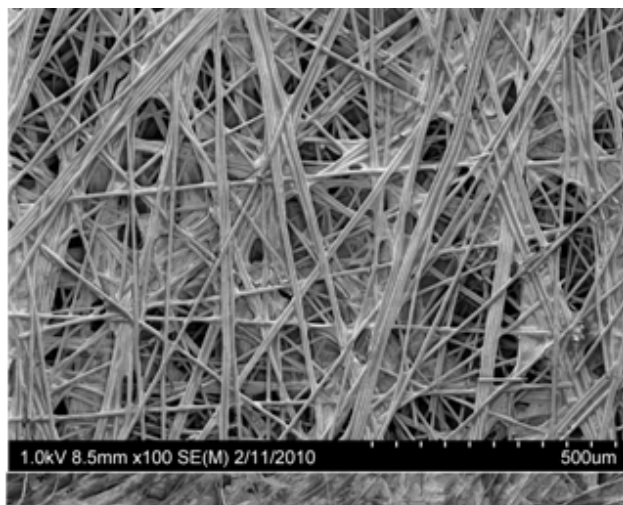

(b)

(c)

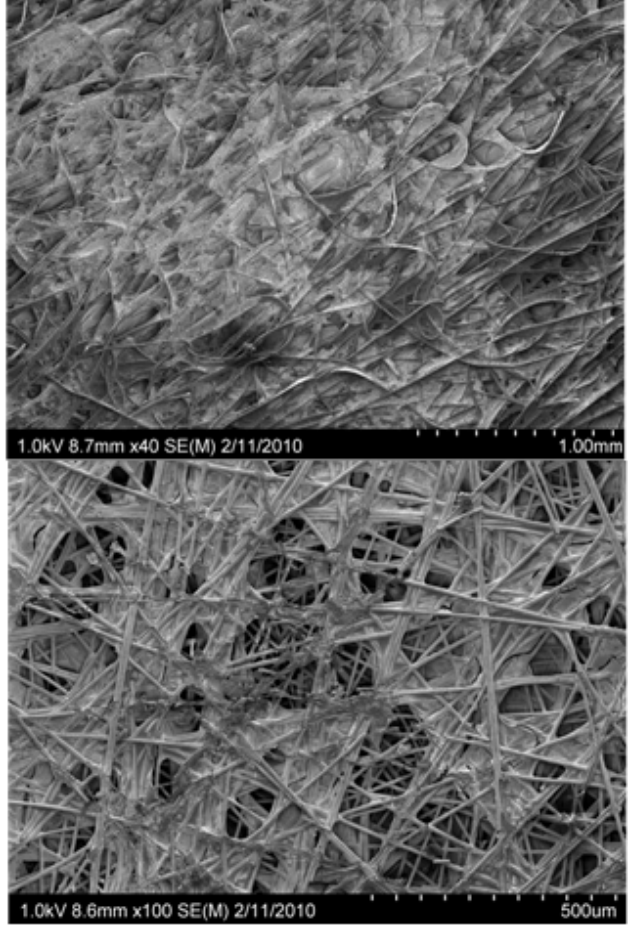

Figure 1: SEM Images: a) TGP 120 with 5\% PTFE content; b) SGL Sigracet 10AA; c) compressed TGP 120 with 5\% PTFE content.

The porosity of the samples which was reported by manufacturer and Gostick et el. [16] are used in the present study. Fiber diameter was reported by Gostick et al. [16] and was also independently calculated using scanning electron microscopy (SEM) images. The thickness of the samples was measured using a micrometer; the maximum uncertainty associated with the measurements is less than $10 \mu \mathrm{m}$. The properties of the purchased samples are listed in Table 1. 


\section{$2.2 \quad$ Test apparatus}

An air permeability test bed was designed and built for measuring the through-plane permeability, see Figure 2. The high pressure air was supplied by a high pressure air tank and controlled using a digital air pressure regulator; the output pressure of the air regulator valve was set to 6 psi during all experiments. Two aluminum blocks were machined and drilled with a $25 \mathrm{~mm}$ diameter hole. The blocks were used as sample holders and GDL samples were sandwiched between the blocks. An O-ring was used between the two aluminum blocks to prevent air leakage from the test section. The up and down stream air flow were connected to a differential pressure transducer, PX277-01D5V, supplied by Omega (Omega Inc., Laval, QC, Canada). The pressure transducer was connected to a PC, using a DAQ purchased from Omega (Omega Inc., Laval, QC, Canada), where the pressure drop values were recorded. The accuracy of the pressure transducer was $1 \%$ of its full scale measuring range (0-1 inch of water). The air flow rate passing through tested GDLs was measured using a flow meter provided by Omega (Omega Inc., Laval, QC, Canada). The accuracy and the range of the air flow meter was 3\% and 0-10 lpm, respectively.

The carbon papers were cut into circular samples of $3 \mathrm{~cm}$ diameter for the experiments. To investigate the compression effects, GDL samples were compressed using a GUNT WP 300 Universal
Material Tester for $15 \mathrm{~min}$; the maximum applied compressive force was less than $20 \mathrm{kN}$ (30 MPa). The thickness of the compressed samples was measured, using the same micrometer, after the load was released to determine the variation in its thickness.

\subsection{Data analysis}

Gas permeability, $K$, is defined using Darcy equation [30]:

$$
-\frac{\Delta P}{t}=\frac{\mu}{K} U_{D}
$$

where $U_{D}$ is the volume averaged velocity through porous media, $\mu$ is viscosity, $\Delta P$ is the pressure drop across the sample, and $t$ is the GDLs thickness. Equation (1) is valid for incompressible, steady, constant properties, single-phase (no-surface tension forces), and low Reynolds number flows. The Reynolds number based on the fiber diameter, $\operatorname{Re}=\rho U_{D} d / \mu$, was lower than 0.1 in the present study; therefore, the Darcy equation was applicable for analyzing the experimental results. The maximum measured pressure drop across the samples was less than $200 \mathrm{~Pa}$. As such, it was assumed that the compressibility effects for such small values of pressure drop can be negligible and the Darcy equation in the form of Eq. (1) was applicable in the present study. $U_{D}$ was calculated by dividing the volumetric flow rate by the sample cross-sectional area.

Table 1: Physical properties of different carbon papers used in the present study: porosity and PTFE content provided by manufacturer; the measured thicknesses and fiber diameters.

\begin{tabular}{lcccc}
\hline \hline \multicolumn{1}{c}{ GDL } & $\begin{array}{c}\text { Thickness } \\
(\mu \mathrm{m})\end{array}$ & Porosity $^{*}$ & $\begin{array}{c}\text { Fiber diameter } \\
(\mu \mathrm{m})\end{array}$ & $\begin{array}{c}\text { PTFE content } \\
(\%)\end{array}$ \\
\hline SGL-Sigracet 10AA & $390-410$ & 0.88 & 9.2 & 0 \\
TGP-H-90 & $255-270$ & 0.79 & 9.3 & $0-30$ \\
TGP-H-120 & $370-385$ & 0.79 & 9.3 & 0 \\
\hline \hline
\end{tabular}

${ }^{*}$ The porosity is reported for GDLs with 0\% PTFE content. 
(a)

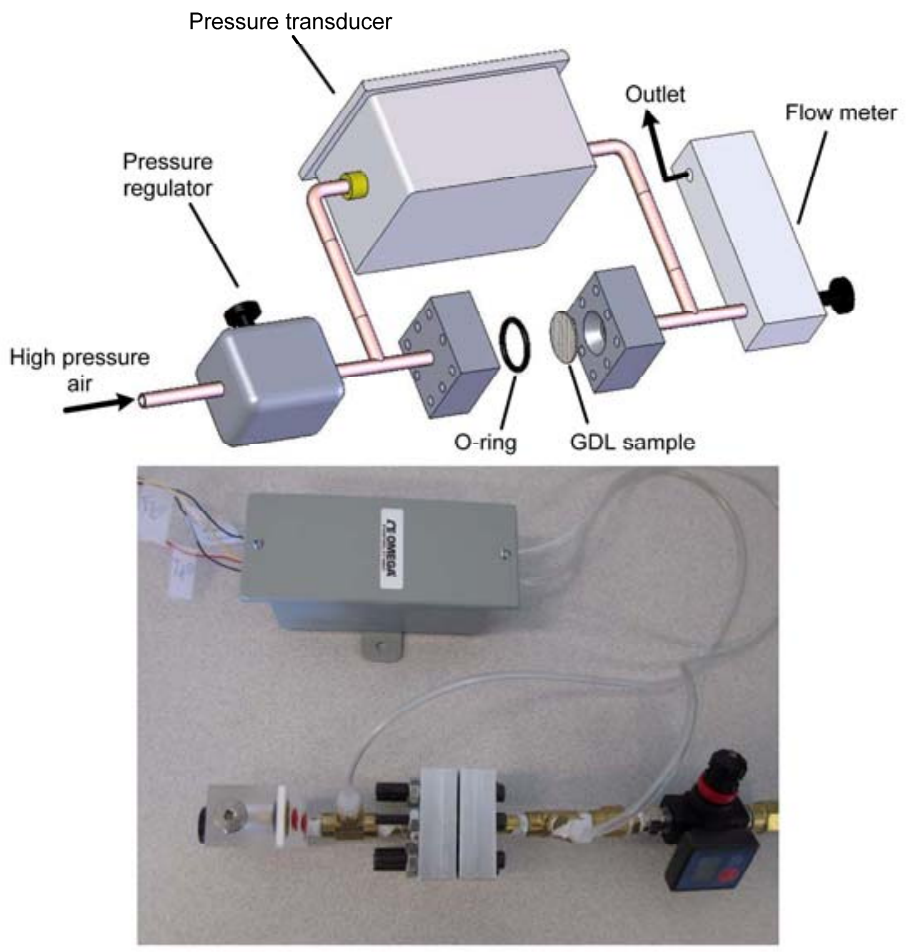

Figure 2: The air permeability test bed: a) schematic of the apparatus (exploded view) b) actual test setup.

Assuming Darcy’s law in a porous structure implies a linear relationship between the pressure drop and the fluid velocity in the media. As an example, this linear relationship can be observed in the experimental results for samples of compressed TGP-H-120 in Figure 3 which justifies Darcy's assumptions.

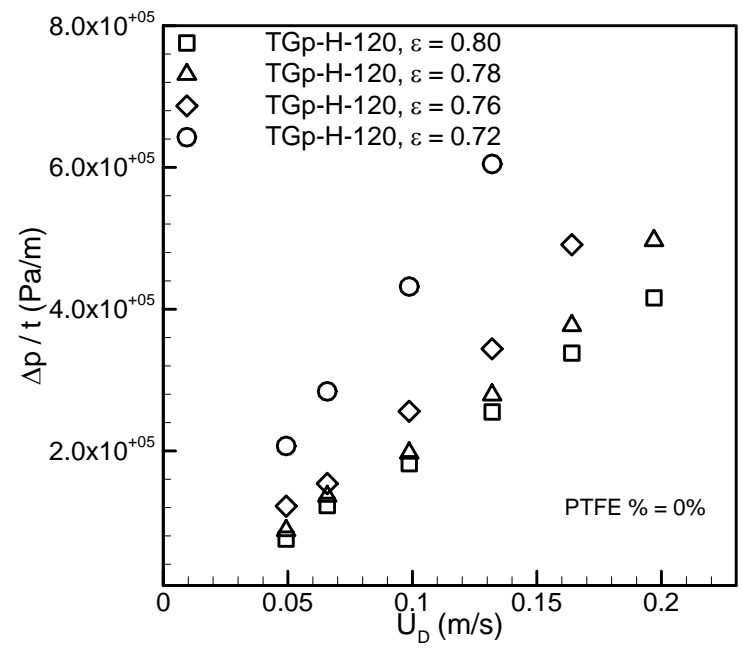

Figure 3: Measured pressure drops for samples of compressed TGP-H-120.
The measured values of pressure drop, $\Delta P_{\text {total }}$, during the experiment were:

$$
\Delta P_{\text {total }}=\Delta P_{\text {sample }}+\Delta P_{\text {min }}
$$

where $\Delta P_{\text {sample }}$ is the pressure drop associated within the sample and $\Delta P_{\min }$ is the pressure drop related to minor losses in the testbed, i.e., exit and entrance section of the sample holder . To account for the minor pressure losses, the pressure drop in the test section was measured once without any GDL samples to determine $\Delta P_{\min }$ for the entire range of flow rate considered in the present experimental work.

The uncertainty associated with the through-plane permeability, calculated based on the measured variables using the Darcy's equation, can be estimated from [31]:

$$
\frac{E(K)}{K}=\sqrt{\left(\frac{E(t)}{t}\right)^{2}+\left(\frac{E(\Delta P)}{\Delta P}\right)^{2}+\left(\frac{E(Q)}{Q}\right)^{2}}
$$

where $E($.$) is the uncertainty in the measurement of$ each parameter; these values are listed in Table 2. 
The maximum uncertainty in the calculated values of permeability is estimated to be $9 \%$.

Table 2: Uncertainty values for measured parameters.

\begin{tabular}{ll}
\hline \hline Parameter & Uncertainty \\
\hline$t$ & $10 \mu m$ \\
$\Delta P$ & $1 \%$ of full scale \\
$Q$ & $3 \%$ of full scale \\
$\varepsilon$ & $5 \%$ \\
$K$ & $9 \%$ \\
\hline \hline
\end{tabular}

\section{Theoretical Model}

Tamayol and Bahrami (TB) [29] employed a scale analysis and offered a scale for permeability of planar fibrous microstructure, shown in Figure 4, as:

$$
K \sim \varepsilon \delta_{\min }^{2} \tau
$$

where $\varepsilon$ was the porosity, $\tau$ was the tortuosity factor, and $\delta_{\min }$ was half of the minimum opening between two adjacent fibers $(S-d)$, see Figure 4 . They estimated the tortuosity factor from the Bruggeman equation [32]:

$$
\tau=\left(\frac{1}{\varepsilon}\right)^{0.5}=\left(\frac{1}{1-\varphi}\right)^{0.5}
$$

where $\varphi$ was the solid volume fraction, i.e., $\varphi=1-\varepsilon$. Substituting for geometrical parameters, the following relationship was reported for the dimensionless through-plane permeability:

$$
\frac{K}{d^{2}}=0.008 \sqrt{(1-\varphi)}\left[\left(\frac{\pi}{4 \varphi}\right)^{2}-2 \frac{\pi}{4 \varphi}+1\right]
$$

Figure 4: The 2D unit cell considered in the present study.

The constant value in Eq. (6), i.e., 0.008, was found through comparison with experimental data collected from different sources for permeability of various planar fibrous materials. Hao and Cheng [23] numerically calculated the tortuosity factor for carbon papers and proposed the following correlation:

$$
\tau=1+0.72 \frac{1-\varepsilon}{(\varepsilon-0.11)^{0.54}}
$$

Our analysis showed that if the constant value in Eq. (6) is replaced by 0.012 and $\tau$ is calculated from Eq. (7) the resulting equation:

$$
\begin{aligned}
& \frac{K}{d^{2}}=0.012(1-\varphi)\left[\left(\frac{\pi}{4 \varphi}\right)^{2}-2 \frac{\pi}{4 \varphi}+1\right] \\
& {\left[1+0.72 \frac{\varphi}{(0.89-\varphi)^{0.54}}\right]}
\end{aligned}
$$

can predict the experimental data for GDLs more accurately.

To enable the model of Tamayol and Bahrami [29] to accommodate the effects of compression factor and PTFE content, the relationship between these properties and the solid volume fraction should be determined.

Figure 1c shows that mechanical compression does not change the shape of fibers. As such, it can be assumed that during the compression process only the thickness of the original fibrous samples changes while the volume of the solid carbon fiber remains constant. Therefore, the relationship between the solid volume fraction, $\varphi_{\text {comp }}$, of a compressed sample with the original value for an uncompressed carbon paper, $\varphi_{0}$, can be expressed as [23]:

$$
\varphi_{\text {comp }}=\varphi_{0} \frac{t_{0}}{t_{\text {comp }}}
$$

where $t_{\text {comp }}$ and $t_{0}$ are the compressed and uncompressed GDL thicknesses, respectively. If the PTFE is added on the carbon paper GDL, the pore volume is randomly filled by the PTFE. It is postulated that PTFE changes the porosity and some pores in the medium are filled. The final porosity, $\varepsilon_{\text {PTFE }}$, can be expressed approximately as a function of PTFE content $\omega$ [23]:

$$
\varepsilon_{\text {PTFE }}=\varepsilon_{0}-a \frac{\omega\left(1-\varepsilon_{0}\right)}{1-\omega}
$$

where $\varepsilon_{0}$ is the original porosity before PTFE treatment. Hao and Cheng [23] suggested $a=0.9$ as 
the density ratio of the carbon fiber and the PTFE [33]. Employing Eqs. (9) and (10), one can predict effects of PTFE and compression on the permeability on the through-plane permeability of GDLs from TB's model.

\section{$4 \quad$ Results and Discussions}

The permeability of various tested samples is calculated using Darcy's law, Eq. (1). The porosity of the compressed samples and GDLs treated with PTFE are calculated using Eq. (9) and (10), respectively.

The effect of mechanical compression and variation of GDL thickness on the permeability is shown in Figure 5. It can be seen that there is a linear relationships between the ratio of compressed to uncompressed permeability of the measured GDLs and the compression ratio, $t_{\text {comp }} / t_{0}$. The experimental data for the through-plane permeability of compressed GDLs, from the present study or reported by others, are plotted in Figure 6 and compared with the present model, Eq. (8). The comparison of experimental data with the modified TB model shows that proposed model, Eq. (8), captures the trends of experimental data for compressed GDLs. The through-plane permeability of uncompressed TGP-H-90 was measured as $8.7 \times 10^{-8} \mathrm{~m}^{2}$ which is in good agreement with the value of $8.99 \times 10^{-8} \mathrm{~m}^{2}$ reported by Gostick et al. [16].

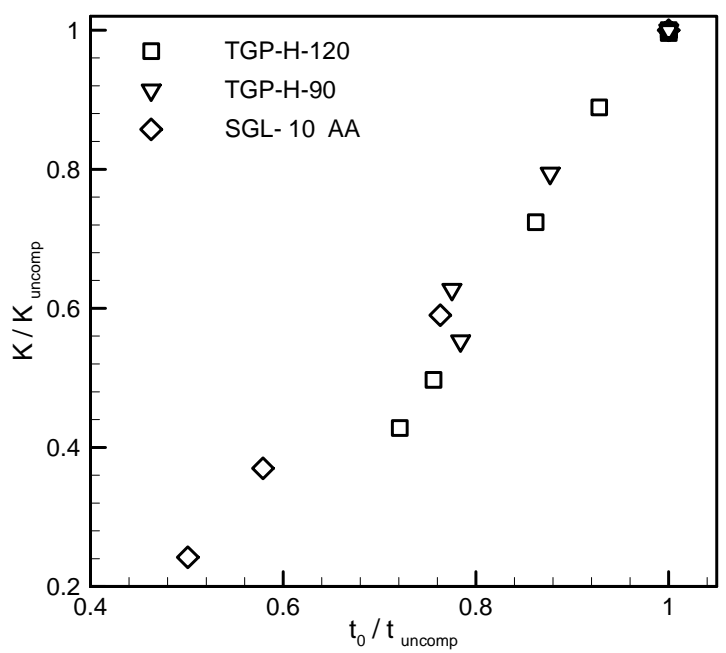

Figure 5: Effect of compression ratio, $t_{\text {comp }} / t_{0}$ on the variation of permeability.

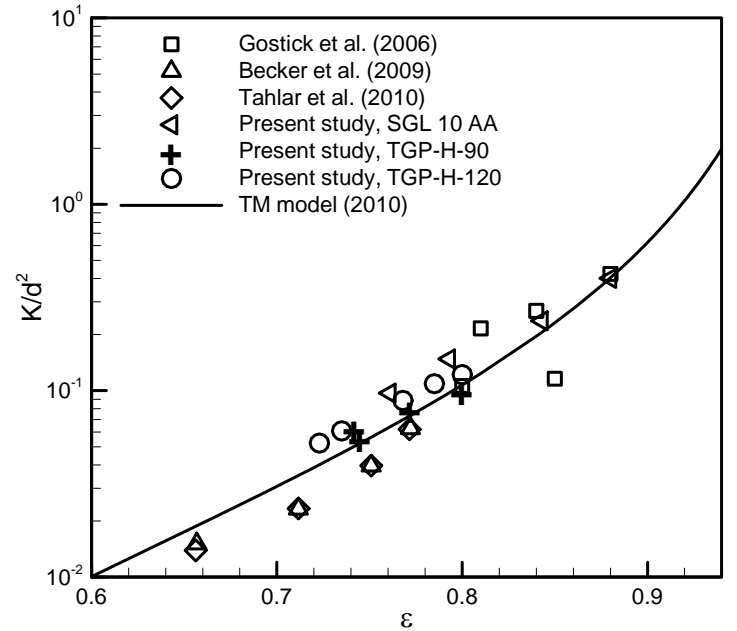

Figure 6: Comparison of the proposed model with the experimental data for compressed GDLs measured in the present study or collected from various sources.

The effect of PTFE content on the through-plane permeability of GDLs is presented in Figure 7. It can be seen that a reverse relationship exists between the PTFE content and the through-plane permeability. The experimental data for TGP-H-120 with various PTFE contents are plotted in Figure 8 and compared with TB model. Porosities of the samples are estimated from Eq. (10). The permeability of the PTFE treated GDLs has a reverse relationship with the PTFE content. It can be seen that Eq. (8) captures the trends of the data. Overall, it can be concluded that the modified TB model can be used in design and optimization of PEMFCs.

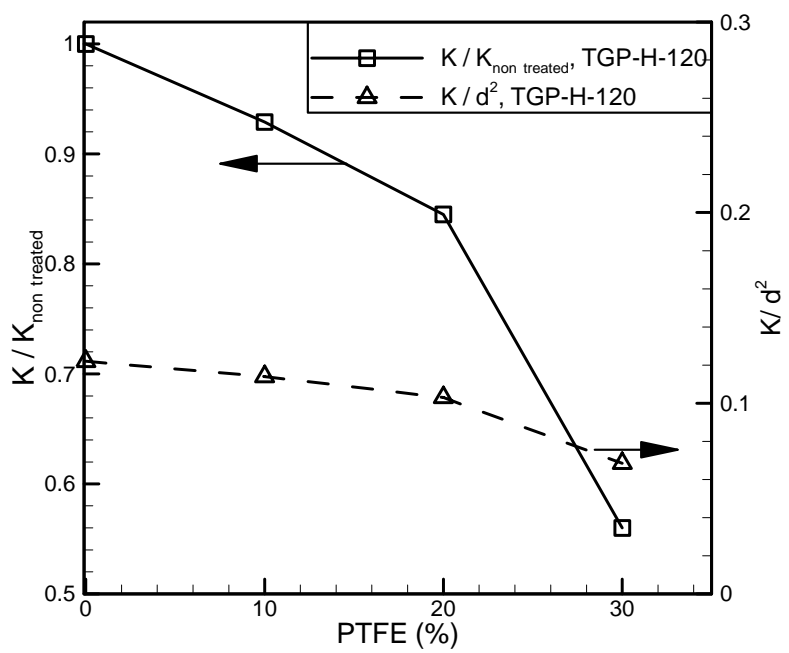

Figure 7: Effect of PTFE content on the throughplane permeability of two set of TGP-H-120 samples with various PTFE contents. 


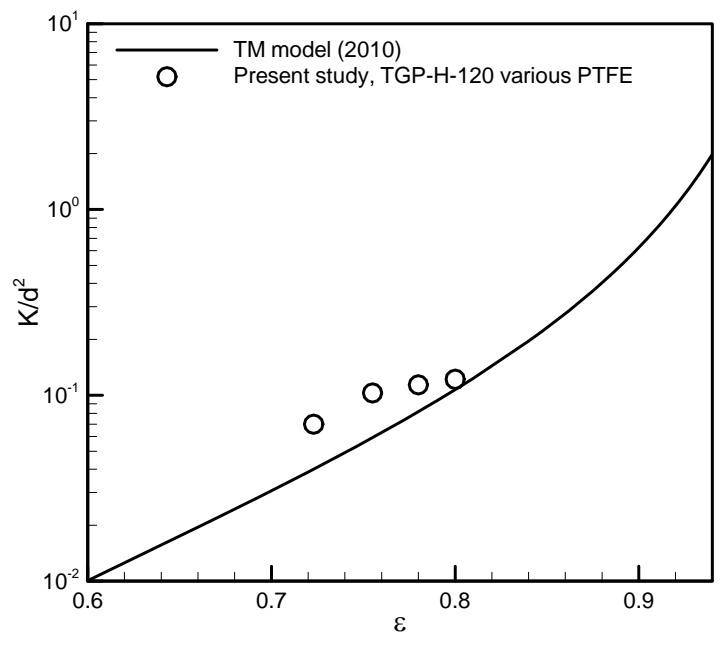

Figure 8: Comparison of the proposed model with the experimental data for TGP-H-120 with various PTFE contents.

\section{$5 \quad$ Conclusions}

An experimental study has been carried out to investigate the effects of compression and PTFE content on the single-phase through-plane permeability of GDLs of PEMFCs. A custom-made testbed was built and several GDL samples were obtained from various manufacturers and tested over a wide range of compression ratios. Moreover, the through-plane permeability of TGP-H-120 carbon papers with various PTFE contents was measured. A scale analysis model originally proposed by Tamayol and Bahrami [29] was modified for predicting the permeability of the PEMFC GDLs. The present model takes into account the PTFE content and compression effects of the permeability.

The highlights of the present study can be summarized as:

- A reverse relationship exists between the through-plane permeability of GDLs and both the compression ratio and PTFE content.

- The revised model of Tamayol and Bahrami, Eq. (8) captures the trends observed in experimental data for permeability of compressed GDLs.

The reported experimental data and the proposed model can be used to guide the design and optimization of PEMFCs, and can be readily implemented into fuel cell models that require specification of the through-plane gas permeability of the GDL.

\section{Acknowledgements}

A.T. would like to thank British Colombia Innovation Council (BCIC) for BCIC Innovation Scholars Award. Financial support of Natural Sciences and Engineering Research Council of Canada, NSERC, is acknowledged.

\section{List of Abbreviations and Variables}

\begin{tabular}{|c|c|}
\hline$d$ & Fiber diameter, $m$ \\
\hline$E()$. & $\begin{array}{l}\text { Uncertainty associated with the } \\
\text { measurement of a parameter }\end{array}$ \\
\hline GDL & Gas diffusion layer \\
\hline$K$ & Permeability, $m^{2}$ \\
\hline$P$ & Pressure, $\mathrm{Pa}$ \\
\hline PEMFC & Polymer electrolyte membrane fuel cel \\
\hline PTFE & Polytetrafluoroethylene \\
\hline$Q$ & Volumetric flow rate, $\mathrm{m}^{3} / \mathrm{s}$ \\
\hline$S$ & $\begin{array}{l}\text { Distance between centers of adjacent } \\
\text { fibers, } m\end{array}$ \\
\hline & Thickness of the samples, $m$ \\
\hline$U_{D}$ & $\begin{array}{l}\text { Volume-averaged superficial velocity, } \\
\mathrm{m} / \mathrm{s}\end{array}$ \\
\hline
\end{tabular}

\section{Greek symbols}

$\begin{array}{ll}\delta_{\text {min }} & \text { The minimum distance between adjacent } \\ & \text { fibers surfaces, } m \\ \varepsilon & \text { Porosity } \\ \mu & \text { Fluid viscosity, } N \mathrm{~s} / \mathrm{m}^{2} \\ \varphi & \text { Solid volume fraction, } \varphi=1-\varepsilon \\ \tau & \text { Tortuosity factor } \\ \omega & \text { PTFE content } \\ \text { Subscript } & \\ \text { comp } & \text { Compressed GDL } \\ 0 & \text { Uncompressed, non-treated GDL }\end{array}$

\section{References}

[1] A. Faghri, Z. Guo, Challenges and opportunities of thermal management issues related to fuel cell technology and modeling, International Journal of Heat and Mass Transfer, 48(19-20) (2005) 38913920.

[2] J. P. Feser, A. K. Prasad, S. G. Advani, Experimental characterization of in-plane permeability of gas diffusion layers, Journal of Power Sources, 162(2) (2006) 1226-1231. 
[3] J. G. Pharoah, On the permeability of gas diffusion media used in PEM fuel cells, Journal of Power Sources, 144(1) (2005) 77-82.

[4] W. W. Sun, B. A. Peppley, K. Karan, Modeling the Influence of GDL and flow-field plate parameters on the reaction distribution in the PEMFC cathode catalyst layer, Journal of Power Sources, 144(1) (2005) 42-53.

[5] J. Ge, A. Higier, H. Liu, Effect of gas diffusion layer compression on PEM fuel cell performance, Journal of Power Sources, 159(2) (2006) 922-927.

[6] J. P. Feser, A. K. Prasad, S.G. Advani, On the relative influence of convection in serpentine flow fields of PEM fuel cells, Journal of Power Sources, 161(1) (2006) 404-412.

[7] G. Hu, J. Fan, S. Chen, Y. Liu, K. Cen, Threedimensional numerical analysis of proton exchange membrane fuel cells (PEMFCs) with conventional and interdigitated flow fields, Journal of Power Sources, 136(1) (2004) 1-9.

[8] M. Khakpour, K. Vafai, Analysis of transport phenomena within PEM fuel cells - An analytical solution, International Journal of Heat and Mass Transfer, 51(15-16) (2008) 3712-3723.

[9] P. T. Nguyen, T. Berning, N. Djilali, Computational model of a PEM fuel cell with serpentine gas flow channels, Journal of Power Sources, 130(1-2) (2004) 149-157.

[10] M. V. Williams, H. R. Kunz, J.M. Fenton, Influence of convection through gas-diffusion layers on limiting current in PEM FCs using a serpentine flow field, Journal of The Electrochemical Society, 151(10) (2004) A1617-A1627.

[11] T. Berning, N. Djilali, A 3D, multiphase, multicomponent model of the cathode and anode of a PEM fuel cell, Journal of The Electrochemical Society, 150(12) (2003) A1589-A1598.

[12] A. Bazylak, V. Berejnov, B. Markicevic, D. Sinton, N. Djilali, Numerical and microfluidic pore networks: Towards designs for directed water transport in GDLs, Electrochimica Acta, 53(26) (2008) 7630-7637.

[13] A. Tamayol, M. Bahrami, Water permeation through gas diffusion layers of proton exchange membrane fuel cells, in: ASME 2010 International Mechanical Engineering Congress \& Exposition, Vancouver, Canada, 2010.

[14] O. Chapuis, M. Prat, M. Quintard, E. ChaneKane, O. Guillot, N. Mayer, Two-phase flow and evaporation in model fibrous media: Application to the gas diffusion layer of PEM fuel cells, Journal of Power Sources, 178(1) (2008) 258-268.

[15] J. Ihonen, M. Mikkola, G. Lindbergh, Flooding of gas diffusion backing in PEFCs, Journal of The Electrochemical Society, 151(8) (2004) A1152A1161.

[16] J. T. Gostick, M. W. Fowler, M. D. Pritzker, M. A. Ioannidis, L.M. Behra, In-plane and through-plane gas permeability of carbon fiber electrode backing layers, Journal of Power Sources, 162(1) (2006) 228238.

[17] M. V. Williams, E. Begg, L. Bonville, H. R. Kunz, J.M. Fenton, Characterization of gas diffusion layers for PEMFC, Journal of The Electrochemical Society, 151(8) (2004) A1173-A1180.

[18] V. Gurau, M. J. Bluemle, E. S. De Castro, Y. M. Tsou, T. A. Zawodzinski, J.A. Mann, Characterization of transport properties in gas diffusion layers for proton exchange membrane fuel cells: 2. Absolute permeability, Journal of Power Sources, 165(2) (2007) 793-802.

[19] J. Becker, R. Fluckiger, M. Reum, F. N. Buchi, F. Marone, M. Stampanoni, Determination of material properties of gas diffusion layers: Experiments and simulations using phase contrast yomographic microscopy, Journal of The Electrochemical Society, 156(10) (2009) B1175B1181.

[20] D. Tehlar, F. R., A. Wokaun, F.N. Büchi, Investigation of Channel-to-Channel Cross Convection in Serpentine Flow Fields, Fuel Cells, (2010) n/a-n/a.

[21] G. G. Park, Y. J. Sohn, T. H. Yang, Y. G. Yoon, W. Y. Lee, C. S. Kim, Effect of PTFE contents in the gas diffusion media on the performance of PEMFC, Journal of Power Sources, 131(1-2) (2004) 182-187.

[22] M. A. VanDoormaal, J.G. Pharoah, Determination of permeability in fibrous porous media using the lattice Boltzmann method with application to PEM fuel cells, International Journal for Numerical Methods in Fluids, 59(1) (2009) 75-89. [23] L. Hao, P. Cheng, Lattice Boltzmann simulations of anisotropic permeabilities in carbon paper gas diffusion layers, Journal of Power Sources, 186(1) (2009) 104-114.

[24] Y. Shi, J. Xiao, M. Pan, R. Yuan, A fractal permeability model for the gas diffusion layer of 
PEM fuel cells, Journal of Power Sources, 160(1) (2006) 277-283.

[25] G. He, Z. Zhao, P. Ming, A. Abuliti, C. Yin, A fractal model for predicting permeability and liquid water relative permeability in the gas diffusion layer (GDL) of PEMFCs, Journal of Power Sources, 163(2) (2007) 846-852.

[26] A. Tamayol, M. Bahrami, In-plane gas permeability of proton exchange membrane fuel cell gas diffusion layers, in: ASME 2010 3rd Joint USEuropean Fluids Engineering Summer Meeting, Montreal, Canada, 2010.

[27] A. Tamayol, M. Bahrami, Analytical determination of viscous permeability of fibrous porous media, International Journal of Heat and Mass Transfer, 52(9-10) (2009) 2407-2414.

[28] M. M. Tomadakis, T.J. Robertson, Viscous permeability of random fiber structures: comparison of electrical and diffusional estimates with experimental and analytical results, Journal of Composite Materials, 39(2) (2005) 163-188.
[29] A. Tamayol, M. Bahrami, Transverse permeability of fibrous porous media, Physical Review E, (2010).

[30] M. Kaviany, Principles of Heat Transfer in Porous Media, 2nd ed., Springer-Verlag, New York, 1995.

[31] J. R. Taylor, An introduction to error analysis: The study of uncertainties in physical measurements, Second ed., University Science Books,, Sausalito, USA, 1997.

[32] A. Z. Weber, J. Newman, Modeling transport in polymer-electrolyte fuel cells, Chemical Reviews, 104(10) (2004) 4679-4726.

[33] M. F. Mathias, J. Roth, J. Fleming, W. Lehnert, Diffusion media materials and characterization, in: W. Vielstich, H. A. Gasteiger, A. Lamm (Eds.) Handbook of Fuel Cells-Fundamentals, Technology and Applications, John Wiley \& Sons New York, 2003, pp. 517-537. 